\title{
Detection and Study of Cosynthesis of Tetracycline Antibiotics by an Agar Method
}

\author{
By V. DELIĆ AND JASENKA PIGAC \\ 'PLIVA' Pharmaceutical and Chemical Company, Zagreb, Yugoslavia \\ AND G. SERMONTI \\ Institute of Genetics, The University, Palermo, Italy \\ (Accepted for publication 30 August 1968) \\ SUMMARY
}

\begin{abstract}
Pairs of non-tetracycline-producing mutants of Streptomyces rimosus or $S$. aureofaciens were grown side by side on agar. Their ability to produce antibiotic by cosynthesis was tested by placing a strip of agar cut from the combined culture on plates containing Bacillus subtilis. The activity was revealed as an inhibition halo formed on B. subtilis, opposite one or other mutant strain. The strain surrounded by the halo was considered as a converter of an intermediate product secreted by the other strain. Two types of mutants were observed: a rare type probably affecting the main pathway of antibiotic biosynthesis, and a more frequent type probably affecting some regulatory process.
\end{abstract}

\section{INTRODUCTION}

Inactive mutants (that is non-producers of antibiotic) of Streptomyces aureofaciens and $S$. rimosus blocked in the biosynthesis of the tetracycline molecule, have been described by McCormick, Hirsch, Sjolander \& Doerschuk (1960) and by Alikhanian, Orlova, Mindlin \& Zeitzeva (196r). Some pairs of inactive mutants, in mixed liquid culture, were found to produce substantial amounts of tetracycline antibiotics (McCormick et al. 1960). This phenomenon, which is comparable to syntrophism between complementing auxotrophic mutants, has been called 'co-operative biosynthesis' or 'cosynthesis' and it has been attributed to the conversion to tetracycline by one strain of some intermediate synthesized and secreted by the other. The two cosynthesizing strains are blocked in different biosynthetic steps, the 'converter' being blocked at an earlier step than the 'secretor'. The present paper describes a simple method of detecting cosynthesis between pairs of inactive mutants, by an agar plate test, which has the additional advantage of allowing immediate recognition of the converter and secretor member of each pair.

\section{METHODS}

The organisms. Streptomyces rimosus R6, an oxytetracycline producer, and Streptomyces aureofaciens A4, a chlortetracycline producer (both isolated from soil and maintained in the Culture Collection, Department of Industrial Microbiology, Faculty of Technology, Zagreb University) were used for the isolation of inactive mutants. Auxotrophic mutants (methionine (met) and tryptophan (try) requiring) of 
these strains, which had retained their ability to produce antibiotic, were often used as a source of inactive mutants. Streptomyces sp. PLIVA 670, an inactive wild strain isolated from soil, was used in some cosynthesis experiments, together with other wild strains of various origins. Bacillus subtilis 3004 (Culture Collection, Department of Industrial Microbiology, Faculty of Technology, Zagreb University) was used for the bioassay of tetracyclines.

Media. The medium used to test the antibiotic production of the Streptomyces strains was: mal ${ }^{+}$extract $10 \mathrm{~g}$., yeast extract 4 g., glucose $4 \mathrm{~g}$., agar $20 \mathrm{~g}$., tap water $1000 \mathrm{ml}$., pH 6.8. The medium used for $B$. subtilis was: peptone $6 \mathrm{~g}$., caseine hydrolysate 4 g., yeast extract 3 g., beef extract $\mathrm{I} \cdot 5 \mathrm{~g}$., glucose I g., agar $20 \mathrm{~g}$., tap water $1000 \mathrm{ml}$., pH 6.6. All ingredients were Difco standard quality.

Mutagenic treatment. u.v. radiation: agitated suspensions of spores were irradiated by a 'Philips' TUV I5 germicidal lamp, to a survival rate of $\mathrm{I}$ to $0 . \mathrm{I} \%$.

Nitrous acid. Spores were suspended in $0.2 \mathrm{M}$-acetate buffer at $\mathrm{pH} 4.4$. A freshly prepared solution of $0.5 \mathrm{M}-\mathrm{NaNO}_{2}$ was added to the spore suspension to give a final concentration of $0.017 \mathrm{M}$. Treatment was stopped at the required time by diluting samples into five volumes of $0.2 \mathrm{M}$-phosphate buffer $\mathrm{pH} 7 \cdot 0$. After $12 \mathrm{~min}$. of treatment the survival rate was about $0.1 \%$.

Ethyleneimine and 1,3-diepoxybutane. These were added to spore suspensions in water to final concentrations of $0 . I$ and $I \%(v / v)$ respectively. Survival rates of $I$ to $0.1 \%$ were obtained after $60 \mathrm{~min}$. of treatment with ethyleneimine and after $40 \mathrm{~min}$. with I,3-diepoxybutane.

Isolation of inactive mutants. Mutagen-treated suspensions of spores were diluted and spread on agar medium. Colonies picked at random were transferred to a second plate in such a way that not more than Io well separated colonies grew on the second plate. After 7 days' incubation at $28^{\circ}$ melted agar containing spores of Bacillus subtilis was poured over the plates and these were incubated overnight at $37^{\circ}$. Colonies not producing a halo of inhibition were considered as inactive mutants, and were re-tested in liquid medium to prove their inactivity. Inactive mutants were designated otc or $c t c$, according to whether derived from Streptomyces rimosus (oxytetracycline producer) or $S$. aureofaciens (chlortetracycline producer) respectively.

Detection of cosynthesis. Two inactive mutants to be tested were streaked on opposite halves of a plate, about I-2 mm apart (each covering one half of the plate). The plate was incubated 4 to 7 days at $28^{\circ}$. A strip of agar $8 \mathrm{~cm} . \times 0.5$ to $0.7 \mathrm{~cm}$. was cut from the plate at right angles to the line of separation between the two strains. This strip was placed on the surface of an agar plate containing the test organism (B. subtilis). The plate was kept for $2 \mathrm{hr}$ in the refrigerator to allow diffusion of antibiotic from the strip into the plate and then incubated overnight at $37^{\circ}$. Any antibiotic activity was revealed as a zone of inhibition in the growth of $B$. subtilis, around one region of the strip (Pl. I, fig. I).

Paper chromatography. Chromatograms of pieces of agar containing active substances, placed on Whatman no. I paper, were run in nitromethane + benzene + pyridine $(20+10+3$ by vol) and examined under the ultraviolet light. Occasionally, chromatograms were also placed on agar plates containing $B$. subtilis 3004 , kept for $2 \mathrm{hr}$ in a refrigerator, and incubated overnight at $37^{\circ}$. The $R_{\boldsymbol{F}}$ values of products giving an inhibition halo was determined for such chromatograms. Other solvents were also used to check antibiotic substances (see Lancini \& Sensi, 1964). 


\section{RESULTS}

\section{Isolation of inactive mutants}

Table I summarizes the results obtained in a search for inactive mutants of Streptomyces rimosus and $S$. aureofaciens. Inactive mutants represented about 0.2 to $0.5 \%$ of the survivors of mutagenic treatment; the most effective was ultraviolet irradiation with a survival rate of $I$ to $0.1 \%$. Many of the inactive mutants showed a decreased ability to sporulate, or loss or increase of brown pigment production. Some of the mutants retained traces of activity: these were often the most effective in cosynthesis.

Table I. Isolation of inactive mutants from Streptomyces rimosus $R 6$ and Streptomyces aureofaciens A4

\begin{tabular}{|c|c|c|c|c|c|c|}
\hline \multirow[b]{3}{*}{ Mutagen* } & \multicolumn{3}{|c|}{ S. rimosus $\mathrm{R} 6$} & \multicolumn{3}{|c|}{ S. aureofaciens A4 } \\
\hline & \multirow{2}{*}{$\begin{array}{c}\text { No. } \\
\text { colonies } \\
\text { tested }\end{array}$} & \multicolumn{2}{|c|}{$\begin{array}{c}\text { Inactive mutants } \\
\text { isolated }\end{array}$} & \multirow{2}{*}{$\begin{array}{l}\text { No. } \\
\text { colonies } \\
\text { tested }\end{array}$} & \multicolumn{2}{|c|}{$\begin{array}{l}\text { Inactive mutants } \\
\text { isolated }\end{array}$} \\
\hline & & no. & $\%$ & & no. & $\%$ \\
\hline Ultraviolet radiation & 3976 & 22 & 0.55 & 2295 & 4 & 0.30 \\
\hline Nitrous acid & 732 & 0 & 0 & I56I & 3 & 0.19 \\
\hline Ethyleneimine & 509 & I & 0.19 & - & - & - \\
\hline Diepoxybutane & 2694 & 6 & 0.22 & 764 & I & 0.17 \\
\hline
\end{tabular}

As shown in Pl. I, fig. I, a clear zone of inhibition could surround a short region of the strip bearing the two combined strains. Such a halo, which did not appear if the strains had been grown on separate plates, is evidence of antibiotic cosynthesis. As a rule the inhibition occurred only on one side of the line of separation between the mutants. The mutant surrounded by the halo was evidently the one which converted to tetracycline a compound, possibly a normal precursor, secreted by the other mutant. The halo-producing mutant was thus blocked in an earlier step of the biosynthesis. In the example of P1. I, fig. I, an inactive mutant of Streptomyces rimosus (otc C I5) is shown to complete antibiotic biosynthesis started by a wild-type inactive Streptomyces sp. 670. The nature of the antibiotic produced was in some cases tested by paper chromatography of a piece of agar cut from the region of activity. In intraspecific cosynthesis, pairs of $S$. rimosus mutants always produced oxytetracycline, and S. aureofaciens mutants, chlortetracycline.

\section{Grouping of inactive mutants of Streptomyces rimosus}

Twenty-eight inactive mutants (otc) of Streptomyces rimosus, all obtained from auxotrophic mutants, were tested for their cosynthetic ability in 756 possible pairs. They were grouped according to their pattern of complementation, into eight groups. Each of the larger group comprised inactive mutants derived from different auxotrophic parents, but inactive mutants from the same auxotrophic parent could be found in different groups. For instance, inactive mutants in group A were obtained from three auxotrophs: $m e t-9$, met-I8, try-2, and inactive derivatives from mutant met-I8 were found in groups $\mathrm{A}, \mathrm{F}$ and $\mathrm{G}$. This suggests that the complementation 
pattern of an auxotrophic inactive mutant was not due to its nutritional requirement. The cosynthetic interactions between mutants of different complementation groups are shown in Table 2, where the code number of the mutants belonging to the various groups is also recorded. Mutants of groups A, B, C and E (which have been named class I) show a mutually simple complementation pattern, each mutant giving cosynthetic activity with all mutants of the other groups. More complex patterns are shown by mutants of the groups $F, G, H$ and $D$ (called class 2) which complement with two (F), or only one ( $G$ and $H)$, or none (D) of the other groups of mutants (Table 2). Mutants of class 2 never act as 'converters', as is evident from Table 2, where letters corresponding to groups $F, G, H$ and $D$ never appear in the diagram illustrating cosynthesis between groups. Consequently members of these four groups never complement each other. In this respect group $\mathbf{A}$ could be placed in class 2 . Class I groups can be arranged in order, by placing each secretor mutant after all mutants converting its product. They turn out to be in the sequence: $\mathrm{E} \rightarrow \mathrm{C} \rightarrow \mathrm{B} \rightarrow \mathrm{A}$. Class 2 groups give complementation, if any, only with the first two groups of this sequence.

Table 2. Grouping of inactive mutants of Streptomyces rimosus $R 6$

Attribution of mutants to complementation groups

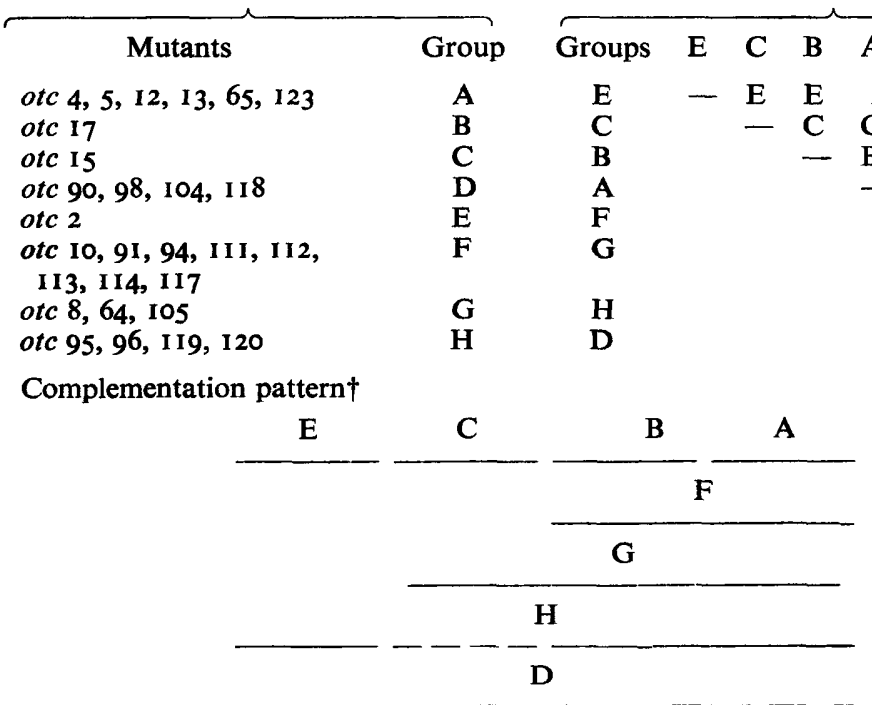

* The sign - indicated no cosynthesis. Each letter indicates cosynthesis with a halo on the side of the strain of the corresponding group.

$\dagger$ Non-overlapping segments correspond to complementing groups.

\section{Grouping of inactive mutants of Streptomyces aureofaciens}

Ten inactive mutants of Streptomyces aureofaciens (ctc) were studied in combined cultures, all fully inactive derivatives of prototrophic strain. Cosynthesis was barely evident with many pairs and reliable evidence was obtained only after repeated tests. Some of the mutants (ctc 8, ctc 9, and $c t c$ 10) had to be disregarded because of doubtful responses. The remaining six were placed in four groups, the complementation 
pattern of which is represented in Table 3. Groups A, B and C belong to class I (see preceding section), group D to class 2.

The order of the steps controlled by mutants of class I was deduced by distinguishing the 'secretor' and 'converter' member of each pair. Mutants $c t c A r, c t c B 3$ and ctc $C 8$ were particularly considered (Pl. I, fig. 2). The three groups could be arranged in the order $\mathrm{C} \rightarrow \mathrm{B} \rightarrow \mathrm{A}$ (i.e. $5 \rightarrow 3 \rightarrow \mathrm{I}$ ).

Table 3. Grouping of inactive mutants of Streptomyces aureofaciens A4

\begin{tabular}{|c|c|c|c|c|c|c|}
\hline \multicolumn{2}{|c|}{$\begin{array}{l}\text { Attribution of mutants to } \\
\text { complementation groups }\end{array}$} & \multicolumn{5}{|c|}{ Cosynthesis between groups* } \\
\hline Mutants & Group & Groups & $\mathbf{A}$ & B & C & D \\
\hline $\begin{array}{l}\text { ctc } 1,2,4 \\
\text { ctc } 3 \\
\text { ctc } 5 \\
\text { ctc } 6,7 \\
\text { ctc } 8,9 \text {, } 10\end{array}$ & $\begin{array}{c}\text { A } \\
\text { B } \\
\text { C } \\
\text { D } \\
\text { Doubtful }\end{array}$ & $\begin{array}{l}\text { A } \\
\text { B } \\
\text { C } \\
\text { D }\end{array}$ & - & B & $\begin{array}{l}\text { C } \\
\mathbf{C}\end{array}$ & $\begin{array}{l}\text { A } \\
\text { - }\end{array}$ \\
\hline
\end{tabular}

Complementation pattern: $\uparrow$

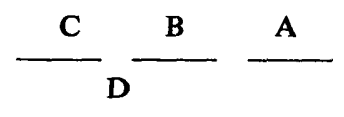

* See note* to Table $2 . \quad$ † See note $\uparrow$ to Table 2.

Detection of interspecific cosynthesis. Cosynthetic activity was also evident in combinations between an inactive mutant of Streptomyces rimosus and an inactive mutant of $S$. aureofaciens. Two mutants of $S$. rimosus belonging to groups otc $E$ and otc $B$, combined with a mutant of group ctc $A$ of $S$. aureofaciens, gave a halo on the side of the $S$. rimosus mutants, which thus acted as converters. This result was expected, because gene $\operatorname{ctc} A$ is a late gene in chlortetracycline biosynthesis, while otc $B$ and otc $E$ are earlier genes in oxytetracycline biosynthesis.

Cosynthesis was also detected between several inactive wild Streptomyces isolates and inactive mutants of either Streptomyces rimosus or $S$. aureofaciens. In fact the best haloes were often observed in such interspecific combinations. The halo invariably appeared on the side of the inactive mutant, the wild-type strain thus acting as a class 2 mutant. Cosynthesis of tetracycline antibiotics in combinations of different streptomycetes with inactive mutants of tetracycline producing species has been reported by McCormick, Sjolander \& Hirsch (196I).

\section{DISCUSSION}

The agar method for the study of tetracycline cosynthesis, which may possibly be of general application in the study of secondary metabolism, has two distinct advantages over liquid mixed culture: the saving of space and material and the immediate recognition of the 'secretor' and the 'converter' member of each pair of cosynthesizing strains. Cosynthesis on agar must presumably involve the diffusion of some product, possibly a normal precursor of tetracycline biosynthesis, since no contact between the organisms was established in the combined culture.

The experiments described, which were carried out primarily to develop the method, have already shown interesting results. The complementation pattern is rather similar in the two species examined. In both, two distinct classes of inactive mutants occurred. 
The first class, comprising groups (genes?) otc $B$, otc $C$, and itc $E$ (and perhaps otc $A$ ) of Streptomyces rimosus, and groups cte $B$ and ctc $C$ (and perhaps $c t c A$ ) of $S$. aureofaciens, consist of groups of mutants which complement with each other, and may act in different combinations either as 'secretors' or as 'converters'. They are very likely mutants in structural genes involved in the main pathway of antibiotic biosynthesis. A second class of mutants, comprising groups otc $D$, otc $F$, otc $C$ and otc $H$ (and perhaps otc $A$ ) of $S$. rimosus and ctc $D$ (and perhaps $c t c A$ ) of $S$. aureofaciens, consists of groups of mutants which do not complement with each other, but complement with only some (if any) of the mutants of the first class, and can only act as secretors in cosynthesis. The nature of these mutants is not clear. The possibility that they arose by multisite mutations covering several structural genes seems unlikely, mainly because of their exceedingly high frequency, as compared with that of the assumed point mutations in the structural genes. They cannot involve genes controlling nondiffusible products, because they could never act (on this assumption) as secretors. It seems more probable that mutants of the second class are not altered in genes directly involved in the main pathway of antibiotic biosynthesis, but in regulatory genes or more generally, genes controlling the onset of secondary metabolism (Bu'lock, 1965), i.e. the shift of normal metabolic channels towards the antibiotic pathway. Whatever the function of genes affected in class 2 mutants, these mutants represent the great majority of the inactive mutants (up to $90 \%$ in S. rimosus, if we regard the otc $A$ ) group as belonging to class 2 ). We can thus conclude that only a small fraction of inactive mutants (class I) are actually blocked in the main antibiotic pathway and only these should be used for the study of pathways of antibiotic biosynthesis.

It is a pleasure to acknowledge the advice of Dr D. Vlašic and to thank him for many helpful discussions. We are also indebted to Miss Jasenka Korajlija for her excellent technical assistance.

\section{REFERENCES}

Alikhanian, S. I., Orlova, N. V., Mindlin, S. Z. \& Zeitzeva, Z. M. (1961). Genetic control of oxytetracycline biosynthesis. Nature, Lond. $189,939$.

Bu'Lock, J. D. (1965). The Biosynthesis of Natural Products: an Introduction to Secondary Metabolism. London: McGraw-Hill Publ. Co. Ltd.

LANCINI, G. C. \& SENSI, P. (1964). Isolation of 2-acetyl-2-decarboxamido tetracycline from culture of Streptomyces psammoticus. Experientia 20, 83.

McCormick, J. R. D., Hirsch, U., Sjolander, N. O. \& Doerschuk, A. P. (1960). Cosynthesis of tetracycline by pairs of Streptomyces aureofaciens mutants. J. Am. chem. Soc. 82, 5006.

McCormick, J. R. D., Sjolander, N. O. \& Hirsch, U. (I96I). Production of tetracyclines. U.S. Patent 2,998,352.

\section{EXPLANATION OF PLATE}

Fig. I. Antibiotic cosynthesis by a pair of inactive Streptomyces strains as revealed by the agar method (central strip). The central strip was cut from a 6-day culture on an agar dish, half seeded with $S$. rimosus otc $C 15$ (darker part of the strip) and half seeded with wild Streptomyces sp. 670. The righthand strip is from a control pure culture of $S$. rimosus otc $C$ 15, and the left-hand strip from a control culture of Streptomyces sp. 670. The strips were placed on agar medium embedded with Bacillus subtilis and the dish was incubated overnight. An inhibition halo is evident around the central strip on the $S$. rimosus half.

Fig. 2. Cosynthesis between inactive mutants of Streptomyces aureofaciens: ctc A I (I), ctc B 3 (3), ctc $C 5(5)$. Strain I is always a 'converter', strain 5 always a 'secretor', strain 3 is a 'converter' with 1 and a secretor with 5 . 
Journal of General Microbiology, Vol. 55, No. I

Plate I

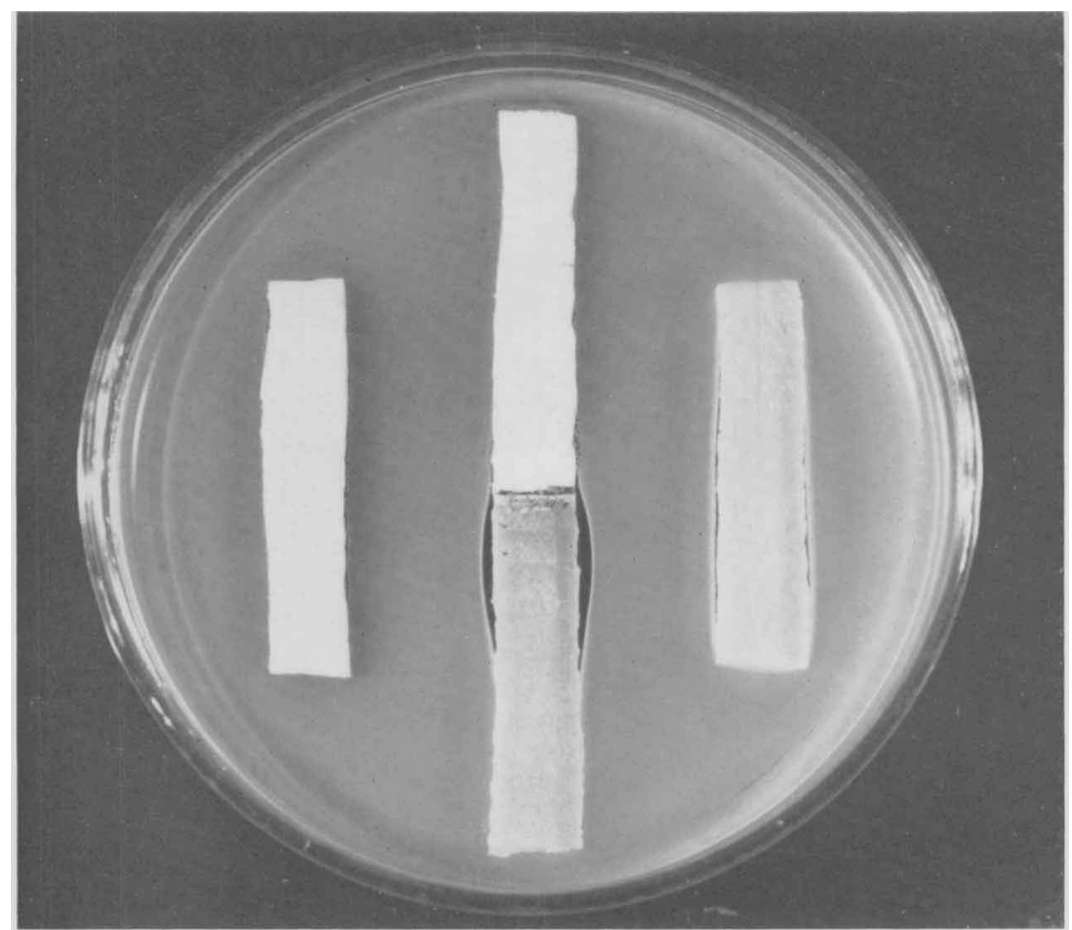

Fig. I

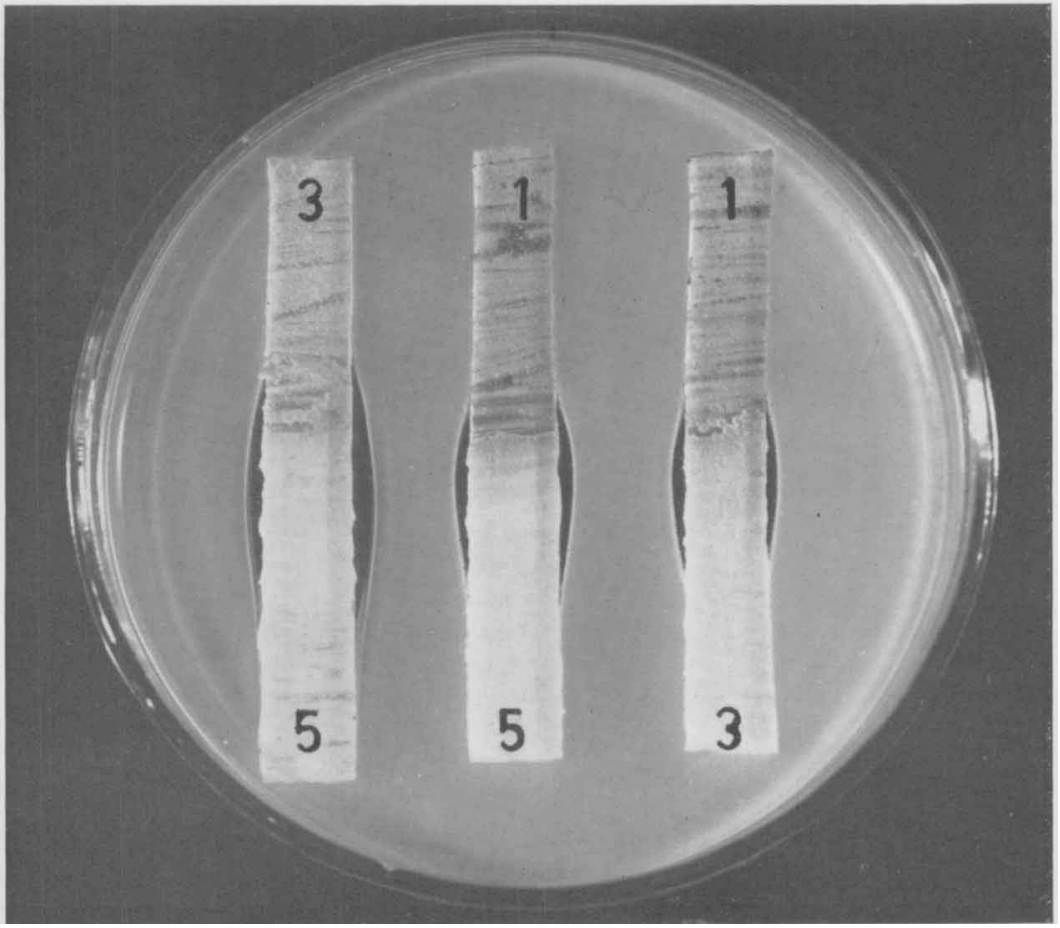

Fig. 2 